\title{
Risk Factors and Clinical Outcomes in Patients with IBD with Melanoma
}

\author{
Loes H. C. Nissen, MD, ${ }^{*}$ Marieke Pierik, $P h{ }_{,}{ }^{+}$Lauranne A. A. P. Derikx, PhD, ${ }^{*}$ Elke de Jong, PhD, ${ }^{\neq}$ \\ Wietske Kievit, PhD, ${ }^{\S}$ Tim R. A. van den Heuvel, $\mathrm{MSc}_{1}^{+}$Alexander R. van Rosendael, MD,"

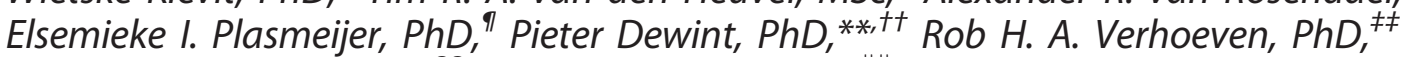 \\ Lucy I. H. Overbeek, PhD, ${ }^{\S \S}$ Iris D. Nagtegaal, PhD, III Frank Hoentjen, PhD, ${ }^{*}$ and \\ Andrea E. van der Meulen-de Jong, PhD" On behalf of Dutch Initiative on Crohn and Colitis, PALGA group \\ and IBD and melanoma group
}

\begin{abstract}
Background: Patients with inflammatory bowel disease (IBD) are at increased risk to develop malignant melanoma and this risk may increase with use of anti-tumor necrosis factor (TNF) therapy. Impaired survival of immunosuppressed melanoma patients is reported in transplant and rheumatology patients. This study aims to (1) identify risk factors for melanoma development in patients with IBD, (2) compare clinical characteristics of melanoma in patients with IBD to the general population, and (3) assess the influence of immunosuppressive medication on survival.
\end{abstract}

Methods: We retrospectively searched the Dutch Pathology Database to identify all Dutch patients with IBD with cutaneous melanoma between January 1991 and December 2011. We then performed 2 case-control studies. To identify risk factors for melanoma development in IBD, we compared patients with IBD with melanoma to the general IBD population. To compare outcome and survival after melanoma diagnosis, we compared cases with non-IBD melanoma patients.

Results: We included 304 patients with IBD with melanoma, 1800 IBD controls, and 8177 melanoma controls. IBD cases had more extensive IBD (ulcerative colitis: pancolitis: cases $44.5 \%$ versus IBD controls without melanoma $28.1 \% ; P<0.01$; Crohn's disease: ileal and colonic disease: cases $57.9 \%$ versus controls $48.9 \% ; P=0.02$ ). Despite a lower Nodes $(\mathrm{N})$-stage in patients with IBD $(\mathrm{N} 1+8.3 \%$ versus $18.2 \% ; P<0.01)$ with comparable Tumor (T) and Metastasis (M) stages, survival was similar between groups, regardless of immunosuppressive or anti-TNF therapy.

Conclusions: This study showed that IBD extent is a risk factor for melanoma development. Despite the lower N-stage in patients with IBD, we could not confirm impaired survival after melanoma in patients with IBD, regardless of anti-TNF and/or thiopurine use.

(Inflamm Bowel Dis 2017;23:2018-2026)

Key Words: inflammatory bowel diseases, melanoma, immunosuppressive therapy

P atients with inflammatory bowel disease (IBD) are at increased risk to develop nonmelanoma skin cancer (also called keratinocyte carcinoma), ${ }^{1-5}$ especially those on thiopurine therapy. ${ }^{3,6,7}$ In addition, a recent meta-analysis showed that the risk to develop cutaneous melanoma is slightly elevated in patients with IBD compared with the general population. ${ }^{2,8}$ Anti-tumor necrosis factor (TNF) therapy may further increase the risk of melanoma development in patients with $\mathrm{IBD},{ }^{3}$ as is the case in rheumatic arthritis patients on anti-TNF therapy. ${ }^{9}$ Thiopurine therapy does not seem to affect the risk of melanoma development in IBD. ${ }^{3}$

Melanoma is considered an immunogenic malignancy: melanoma tumor antigens can trigger the immune response.

Received for publication March 3, 2017; Accepted May 10, 2017.

From the *Department of Gastroenterology and Hepatology, Inflammatory Bowel Disease Center, Radboud University Medical Center, Nijmegen, the Netherlands; ${ }^{\dagger}$ Department of Gastroenterology and Hepatology, Maastricht University Medical Center, Maastricht, the Netherlands; ${ }^{\star}$ Department of Dermatology, Radboud University Medical Center, Nijmegen, the Netherlands; ${ }^{{ }^{R}}$ Radboud Institute for Health Sciences, Radboud University Medical Center, Nijmegen, the Netherlands; ${ }^{\prime}$ Department of Gastroenterology and Hepatology, Leiden University Medical Center, Leiden, the Netherlands; "Department of Dermatology, Leiden University Medical Center, Leiden, the Netherlands; **Department of Gastroenterology and Hepatology, Maasstad Ziekenhuis, Rotterdam, the Netherlands; ${ }^{\dagger \dagger}$ Department of Gastroenterology and Hepatology, MariaMiddelares Ziekenhuis, Gent, Belgium; ${ }^{\star}$ Netherlands Cancer Registry/Netherlands Comprehensive Cancer Organization, Eindhoven, the Netherlands; ${ }^{\S}$ Stichting PALGA,

Houten, the Netherlands; and "III Department of Pathology, Radboud University Medical Center, Nijmegen, the Netherlands.

The authors have no conflict of interest to disclose.

Members of the PALGA group and IBD and melanoma group are listed in the Appendix 1.

Address correspondence to: Loes H. C. Nissen, MD, Department of Gastroenterology and Hepatology, Inflammatory Bowel Disease Center, Radboud University Medical Centre, PO Box 9101, Code 455, 6500 HB Nijmegen, the Netherlands (e-mail: loes.nissen@radboudumc.nl).

Copyright (C) 2017 Crohn's \& Colitis Foundation

DOI 10.1097/MIB.0000000000001191

Published online 22 August 2017. 
Therefore, it is possible that immunosuppression can influence its clinical behavior. Indeed, immunosuppressed patients with cutaneous malignant melanoma were more likely to die of melanoma compared with immunocompetent controls. ${ }^{10-12}$ Overall and melanoma cause-specific 3-year survival were significantly worse in immunosuppressed patients, especially with increasing Breslow thickness. $^{13}$

In patients with IBD, additional risk factors for melanoma are unknown. Furthermore, the clinical course of melanoma in patients with IBD remains unclear, as well as the influence of immunosuppressive IBD therapy on the survival. Therefore, we designed a case-control study with 2 specific aims. First, we aimed at identifying IBD-specific risk factors for melanoma by comparing patients with IBD with melanoma to patients with IBD without melanoma. Second, we researched clinical characteristics and survival after melanoma by comparing patients with IBD with melanoma to unselected "non-IBD" melanoma patients.

\section{MATERIALS AND METHODS}

\section{Study Design}

We conducted 2 retrospective case-control studies in the Netherlands (Fig. 1).

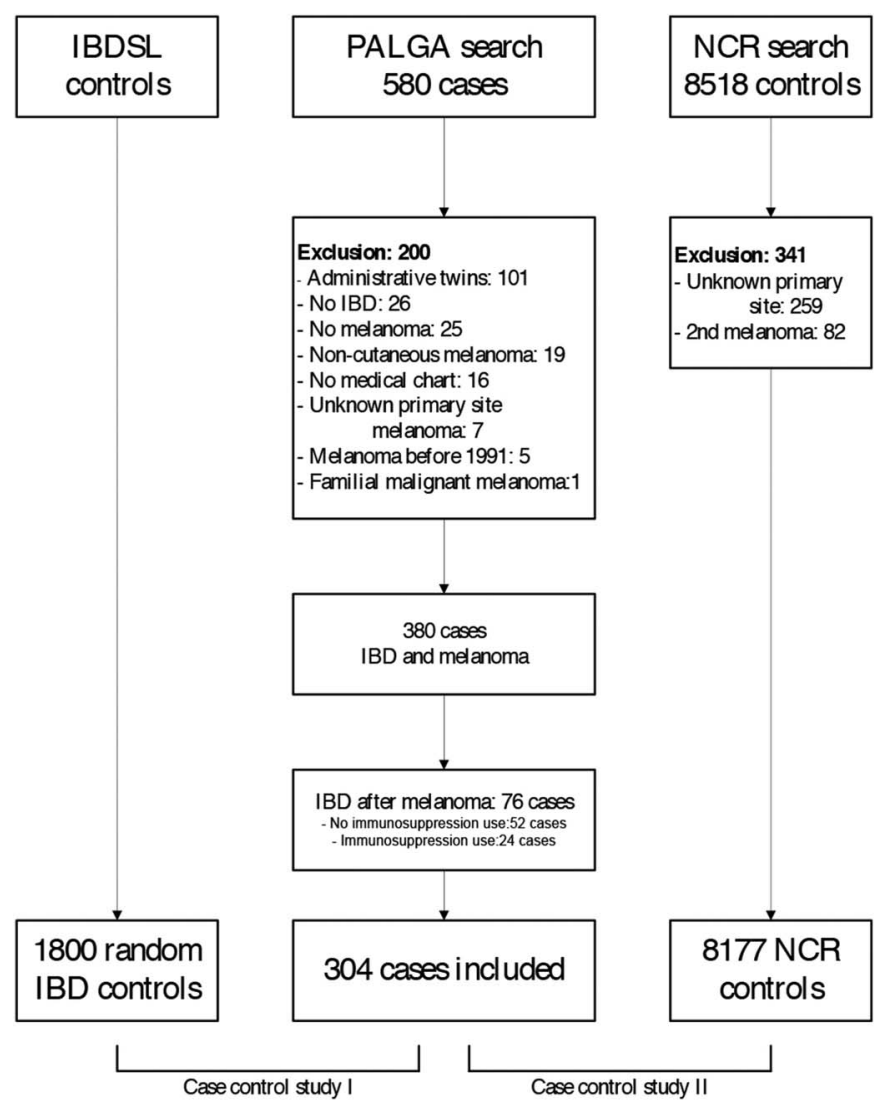

FIGURE 1. Flow chart patient selection. IBDSL, IBD South Limburg cohort; NCR, Netherlands Cancer Registry.
The first case-control study (I) was performed to identify risk factors for melanoma development in patients with IBD. The second case-control study (II) was performed to compare the clinical course after melanoma. This study was approved by the PALGA Privacy Commission and Scientific Council and by the medical ethical committee of the Leiden University Medical Center (number P13.034), the Netherlands.

\section{IBD Case Selection}

All patients diagnosed with both IBD and cutaneous melanoma between January 1991 and December 2011 in the Netherlands were identified using a PALGA-database search. PALGA is the Dutch nationwide network and registry of histoand cyto-pathology, which has complete national coverage for academic and nonacademic hospitals since 1991. ${ }^{14}$ Multiple studies on malignancies in IBD were previously performed using PALGA including gastric cancer, neuro-endocrine tumors, colon cancer, and pouch cancer. ${ }^{15-17}$ In the current study, the following search terms were used: "Crohn's Disease," "Ulcerative Colitis," "Inflammatory Bowel Disease," "terminal ileitis," "regional ileitis," "idiopathic colitis," "chronic idiopathic bowel disease," or "enteritis regionalis" and combined them with "melanoma (primary and metastasis)" or "melanoma in situ." Based on the PALGA search results, anonymized patient charts in all hospitals were evaluated to confirm diagnoses and to collect additional demographic and clinical data. The IBD diagnosis was based on a combination of clinical, endoscopic, histological, and radiographic criteria ${ }^{18}$ and classified according the Montreal classification. ${ }^{19}$

All patients with IBD with primary cutaneous (in situ or invasive) melanoma were included in the study. Patients were excluded when either the IBD or melanoma diagnosis could not be confirmed, the incidence date of the primary melanoma was before 1991 or after 2011, IBD was diagnosed after melanoma diagnosis, or when the patient was known to have familial melanoma syndrome.

\section{Controls Selection}

\section{Case-control Study I: Risk Factor Identification for Melanoma Development in Patients with IBD}

For the identification of melanoma risk factors, 1800 nonmelanoma IBD controls were randomly selected from the IBD South Limburg Cohort (IBDSL Cohort), ${ }^{20}$ based on a 1:3 ratio of the first PALGA search results. No matching was performed as we did not want to exclude any potential risk factors.

The IBDSL cohort is a population-based prospectively followed cohort in which all new cases of adult IBD are enrolled since 1991. South Limburg is an enclosed geographic area in the southeast of the Netherlands with 605,000 inhabitants and 3 hospitals. As cross-border health care is limited and migration rates are low, South Limburg provides a good setting for population-based research. In total 2807 patients with IBD (40.9\% Crohn's disease [CD], 59.0\% ulcerative colitis [UC]) 
are included in the IBDSL Cohort, which represents $>93 \%$ of the IBD population in South Limburg. ${ }^{20}$

Analyses were performed between IBDSL controls and (1) IBD cases with invasive melanoma (2) IBD cases with in situ melanoma and (3) all IBD cases with either invasive melanoma or in situ melanoma, to increase the statistical power. ${ }^{16}$

\section{Case-control Study II: Melanoma Characteristics and Clinical Course in Patients with IBD}

To compare clinical characteristics and outcomes of melanoma between patients with IBD and the general population, non-IBD controls were selected from the Netherlands cancer registration (NCR; managed by the Netherlands Comprehensive Cancer Organisation [NCCO]) from January 1991 until December 2011. Since 1989, the NCR registers the incidence of cancer in the Netherlands, for this study data of the region "Noord-Brabant" and "Noord-Limburg," 2 provinces in the south of the Netherlands, covering an area with 2.3 million inhabitants were used. This specific region was used as in this region data on comorbidity (including IBD) was registered. We used the search terms "melanoma" and "melanoma in situ." Only data on cutaneous (in situ) melanoma were retrieved from the NCR.

\section{Data Collection}

For IBD cases, the following data were anonymously collected: age, sex, medical history including a diagnosis of primary sclerosing cholangitis, alcohol and smoking history, type of IBD based on histopathologic evaluation, date of IBD diagnosis, IBD phenotype according to the Montreal Classification, ${ }^{19}$ use and duration of IBD-related immunosuppressive therapy (corticosteroids, thiopurines, methotrexate, cyclosporin, and anti-TNF therapy), and previous IBD surgery. For IBD controls, the same variables were collected, although alcohol and smoking history were only partially available and duration of immunosuppressive medication was not available.

The following melanoma variables were collected for both cases and controls: year of melanoma diagnosis, localization, histopathologic evaluation, clinical and pathological tumor stage according the TNM classification (fourth edition until 1992; revised fourth edition 1993 until 1998; fifth edition 1999 until 2002; sixth edition 2003 until 2009), Breslow thickness, Clark level, treatment (surgery, chemotherapy [including immunotherapy], and radiotherapy), and overall survival.

IBD cases were anonymously and encrypted linked to the NCR database, for reasons of quality control and completion of collected melanoma data.

\section{Statistical Analyses}

IBD cases were compared with IBDSL and NCR controls using univariable analyses to identify potential risk factors and to compare clinical characteristics. For the univariable analysis, we used a $\chi^{2}$-test or Fisher exact test (if expected cell counts were $<5$ ) for categorical data and independent Student $t$ test or MannWhitney $\mathrm{U}$ test for continuous data. Variables with a $P$ value of
$<0.1$ in the univariable analyses were included in the multivariable analyses. A $P$ value of $<0.05$ was considered statistically significant. All our analyses were performed with IBM SPSS statistics version 20.0 for windows (SPSS Inc., Chicago, IL).

For case-control study I, a binary logistic regression analysis with backward elimination of nonsignificant confounders was performed to determine risk factors for patients with IBD to develop a melanoma. The odds ratios (ORs) were presented with $95 \%$ confidence interval $(95 \% \mathrm{CI})$. This multivariable model was always adjusted for the duration of follow-up (fixed variable), to correct for differences in follow-up between IBD cases and IBDSL controls. For the IBD cases, follow-up was defined as time since IBD diagnosis until melanoma diagnosis. For controls, follow-up was defined as time since IBD diagnosis until death or end of follow-up.

As medication use in especially the distant past might not be reliable and may be different from current regimes, medical therapy was not included in the first multivariable analysis. Therefore, multivariable logistic regression analysis was performed including patients with an IBD diagnosis since 1991 in both the case and control groups (sensitivity analysis). As UC and $\mathrm{CD}$ are classified different according to the Montreal classification, multivariable analysis was performed separately for $\mathrm{UC}$ and $\mathrm{CD}$.

For case-control study II survival plots were derived from Kaplan-Meier curves. Hazard ratios (HRs) were calculated. Confounder correction was performed using a Cox regression model with forward sampling. A covariate was considered as a confounder when the beta coefficient of the variable of interest changed by $10 \%$ or more.

\section{RESULTS}

\section{Selection of Cases and Controls}

Five hundred eighty possible cases of patients with IBD with melanoma were identified (Fig. 1) of which 200 were excluded after careful assessment (Fig. 1). In addition, 76 patients were excluded because IBD was diagnosed after melanoma diagnosis. In total, 304 patients who developed a melanoma after IBD diagnosis were included (57 in situ and 247 invasive melanoma).

For case-control study I, we randomly selected a nonmelanoma control group consisting of 1800 patients with IBD from the IBDSL cohort (Fig. 1).

For case-control study II, the NCR search yielded 8518 non-IBD melanoma patients in the general population (Fig. 1). We excluded 341 patients (259: unknown primary site and 82: second melanoma), resulting in 8177 melanoma patients selected from the general population.

\section{Case-Control Study I: Risk Factor Identification for Melanoma Development in Patients with IBD}

IBD extent differed between IBD cases and IBDSL controls (Table 1): pancolitis was more common in cases in comparison 
TABLE 1. Risk Factors for Melanoma Development

\begin{tabular}{|c|c|c|c|c|c|c|c|c|c|c|}
\hline Variables & $\begin{array}{l}\text { IBDSL, } \\
\mathrm{N}=1800\end{array}$ & $\begin{array}{c}\text { Melanoma } \\
\text { Total, } \mathrm{N}=304\end{array}$ & Missing & $P$ & $\begin{array}{c}\text { Melanoma } \\
\text { In Situ, } \mathrm{N}=57\end{array}$ & Missing & $P$ & $\begin{array}{l}\text { Malig Melanoma, } \\
\qquad \mathrm{N}=247\end{array}$ & Missing & $P$ \\
\hline Age at diagnosis, median $(25 \%-75 \%)$, yrs & $39(28-54)$ & $41(28-57)$ & $0 / 0$ & 0.39 & $47.5(30-62)$ & $0 / 0$ & $0.05^{\mathrm{c}}$ & $39(28-55)$ & $0 / 0$ & 0.97 \\
\hline Female sex, $\mathrm{n}(\%)$ & $983(53.5)$ & $178(58.6)$ & $0 / 0$ & 0.10 & $33(57.9)$ & $0 / 0$ & 0.51 & $145(58.7)$ & $0 / 0$ & 0.12 \\
\hline \multicolumn{11}{|l|}{ Smoking (no), n (\%) (only patients with $\mathrm{CD})^{\mathrm{a}}$} \\
\hline Nonsmoker & $253(37.5)$ & $21(41.2)$ & $122 / 70$ & 0.61 & $4(44.4)$ & $122 / 47$ & 0.67 & $17(40.5)$ & $122 / 57$ & 0.70 \\
\hline$\left(\mathrm{Ex}^{-}\right)$smoker & $421(62.5)$ & $30(58.8)$ & & & $5(55.6)$ & & & $25(59.5)$ & & \\
\hline Primary sclerosing cholangitis, $\mathrm{n}(\%)$ & $13(0.7)$ & $6(3.0)$ & $20 / 104$ & $0.01^{\mathrm{c}}$ & $0(0.0)$ & $20 / 20$ & 0.60 & $6(3.7)$ & $20 / 84$ & $<0.01^{\mathrm{c}}$ \\
\hline \multicolumn{11}{|l|}{ IBD type } \\
\hline $\mathrm{UC}, \mathrm{n}(\%)$ & $1004(55.8)$ & $178(59.5)$ & $0 / 0$ & 0.23 & $32(59.3)$ & $0 / 0$ & 0.61 & $146(59.6)$ & $0 / 0$ & 0.26 \\
\hline $\mathrm{CD}, \mathrm{n}(\%)$ & $796(44.2)$ & $121(40.5)$ & & & $22(40.7)$ & & & $99(40.4)$ & & \\
\hline Indeterminate colitis, n (\%) & $0(0.0)$ & 5 & & & 3 & & & 2 & & \\
\hline \multicolumn{11}{|l|}{$\mathrm{UC}^{\mathrm{b}}$} \\
\hline Proctitis (E1), n (\%) & $243(24.4)$ & $19(11.0)$ & $10 / 0$ & $<0.01^{\mathrm{c}}$ & $2(6.5)$ & $10 / 0$ & 0.07 & $17(12.0)$ & $10 / 0$ & $<0.01^{\mathrm{c}}$ \\
\hline Left-sided colitis (E2), n (\%) & $472(47.5)$ & $77(44.5)$ & & & $18(58.1)$ & & & $59(41.5)$ & & \\
\hline Pan-colitis (E3), n (\%) & $279(28.1)$ & $77(44.5)$ & & & $11(35.5)$ & & & $66(46.5)$ & & \\
\hline \multicolumn{11}{|l|}{$\mathrm{CD}^{\mathrm{b}}$} \\
\hline Ileum (L1), n (\%) & $223(28.1)$ & $19(15.7)$ & $1 / 0$ & $0.02^{\mathrm{c}}$ & $5(22.7)$ & $1 / 0$ & 0.64 & $14(14.1)$ & $1 / 0$ & $0.01^{\mathrm{c}}$ \\
\hline Colon (L2), n (\%) & $183(23.0)$ & $32(26.4)$ & & & $4(18.2)$ & & & $28(28.3)$ & & \\
\hline Ileocolonic (L3), n (\%) & $389(48.9)$ & $70(57.9)$ & & & $13(59.1)$ & & & $57(57.6)$ & & \\
\hline Upper GI (L4) (yes), n (\%) & $65(8.2)$ & $12(10.0)$ & $0 / 1$ & 0.50 & $2(9.1)$ & $0 / 0$ & 0.70 & $10(10 / 2)$ & $0 / 1$ & 0.49 \\
\hline Stricturing (B2), n (\%) & $263(33.0)$ & $46(38.3)$ & $0 / 1$ & 0.25 & $9(40.9)$ & $0 / 0$ & 0.40 & $37(37.8)$ & $0 / 1$ & 0.35 \\
\hline Penetrating (B3), n (\%) & $188(23.6)$ & $42(35.0)$ & $0 / 1$ & $<0.01^{\mathrm{c}}$ & $10(45.5)$ & $0 / 0$ & $0.02^{\mathrm{c}}$ & $32(32.7)$ & $0 / 1$ & $0.05^{\mathrm{c}}$ \\
\hline \multicolumn{11}{|l|}{ Medication } \\
\hline Steroids, n (\%) & $1113(62.2)$ & $169(75.1)$ & $12 / 79$ & $<0.01^{\mathrm{c}}$ & $29(74.4)$ & $12 / 18$ & 0.14 & $140(75.3)$ & $12 / 61$ & $<0.01^{\mathrm{c}}$ \\
\hline Thiopurines, n (\%) & $717(40.2)$ & $104(45.6)$ & $17 / 60$ & 0.47 & $18(42.9)$ & $17 / 15$ & 0.73 & $86(42.6)$ & $17 / 45$ & 0.52 \\
\hline Methotrexate, n (\%) & $95(5.3)$ & $7(3.1)$ & $10 / 79$ & 0.16 & $0(0.0)$ & $10 / 17$ & 0.27 & $7(3.8)$ & $10 / 62$ & 0.37 \\
\hline Cyclosporin, n (\%) & $26(1.5)$ & $7(3.2)$ & $10 / 87$ & 0.08 & $1(2.6)$ & $10 / 19$ & 0.44 & $6(3.4)$ & $10 / 68$ & 0.06 \\
\hline Anti-TNF therapy, n (\%) & $350(19.7)$ & $37(15.2)$ & $25 / 60$ & 0.09 & $5(11.9)$ & $25 / 15$ & 0.21 & $32(15.8)$ & $25 / 45$ & 0.19 \\
\hline IBD-related surgery, no (\%) & $1284(71.3)$ & $206(67.8)$ & $0 / 0$ & 0.21 & $18(32.1)$ & $0 / 0$ & 0.54 & $168(68.9)$ & $0 / 0$ & 0.36 \\
\hline $\begin{array}{l}\text { Duration of follow-up since IBD diagnosis, median } \\
\quad(25 \%-75 \%), y\end{array}$ & $7.00(3-13)$ & $9.00(4-17)$ & $0 / 0$ & $<0.01^{\mathrm{c}}$ & $8.00(3.5-15)$ & $0 / 0$ & 0.12 & $9.00(4-19)$ & $0 / 0$ & $<0.01^{\mathrm{c}}$ \\
\hline $\begin{array}{l}\text { a Only smoking data from patients with } C D \text {. } \\
\text { b } \\
\text { b Classified according to Montreal classification. } \\
\text { c } P<0.05 \text {. } \\
\text { IBDSL, IBD South Limburg cohort (no melanoma cases). }\end{array}$ & & & & & & & & & & \\
\hline
\end{tabular}


with UC controls (44.5\% versus $28.1 \% ; P<0.01$ ). CD was more often located in ileum and colon (cases $57.9 \%$ versus $48.9 \%$; $P=$ $0.02)$ and was more often penetrating $(35.0 \%$ versus $23.6 \%$; $P<$ $0.01)$. Furthermore, primary sclerosing cholangitis was more common in cases $(3.0 \%$ versus $0.7 \% ; P=0.01)$ compared with IBDSL controls. We found no difference in IBD-related surgery and use of thiopurines, anti-TNF therapy, methotrexate, and cyclosporin between cases and controls. However, use of corticosteroids was significantly higher in cases compared with controls: $75.1 \%$ versus $62.2 \%(P<0.01)$.

In the multivariable analysis (Table 2), IBD extent was identified as a risk factor for melanoma development in patients with IBD, both in UC (pancolitis OR: 3.09; 95\% CI, 1.670-5.727) and CD (ileocolonic disease: OR: 1.98; 95\% CI, 1.009-3.882). The sensitivity analysis provided similar results.

In the sensitivity analysis for UC, we found corticosteroid use as risk factor (OR: 1.41-3.72) and anti-TNF use as a protective factor for melanoma development in UC (OR: 0.15-0.88) and CD $(0.27-0.92)$. This difference was mainly attributed to the in situ melanoma, as this effect was not found for invasive melanoma only.

\section{Case-Control Study II: Melanoma Characteristics and Outcome in Patients with IBD}

No differences were found in age at diagnosis, sex, tumor location, tumor histopathology, Breslow thickness and melanoma treatment strategy between the IBD cases and controls with melanoma from the general population (Table 3). Patients with IBD presented with a less advanced $\mathrm{N}$-stage $(\mathrm{N}+8.3 \%$ versus $18.2 \% ; P<0.01$ ), but they had comparable $\mathrm{T}$ and $\mathrm{M}$ stages.

We found no differences in the univariable (Kaplan Meier) survival analyses for all cases $(P=0.42)$, in situ $(P=0.63)$ and malignant melanoma $(P=0.68)$. Additional (multivariable) analyses for males, females, Breslow thickness $>2$ or $4 \mathrm{~mm}$ and IBD diagnosis showed no difference.

We compared IBD cases on immunosuppressive medication after melanoma diagnosis with NCR controls. In the univariable analysis, we found no difference in survival in patients with IBD with melanoma using corticosteroids ( $\mathrm{n}=$ 97; $P=0.93$ ) and a better survival of patients with IBD using anti-TNF (n = 35; $P=0.026$; Fig. 2A) after melanoma diagnosis. In addition, we detected a trend toward improved survival in patients using thiopurines ( $\mathrm{n}=83 ; P=0.058$; Fig. $2 \mathrm{~B}$ ). In the multivariable analysis, we found no difference in survival in patients with IBD using anti-TNF (HR: 0.32; 95\% CI, 0.08-1.27) and thiopurines (HR: 0.72; 95\% CI, 0.37-1.31).

Finally, we compared the survival of IBD cases who did and did not use immunosuppressive medication after melanoma diagnosis. Patients (141 of 269) used immunosuppression after melanoma diagnosis; 23/141 (16.3\%) had recurrent disease, compared with $13 / 128$ patients with IBD (10.2\%) without immunosuppressive medication use after IBD diagnosis $(P=0.14)$.

Univariable, we found no difference in survival for patients using corticosteroids $(P=0.41)$, a trend toward better survival in
TABLE 2. Final Model of Binary Logistic Regression Analysis

Odds Ratio $(95 \%$

Model Variable CI)

All melanoma

$\mathrm{UC}($ all cases $=178)$

$\mathrm{CD}($ all cases $=121)$

Montreal E3 disease

$3.09(1.67-5.73)$

Montreal L2 disease

$2.62(1.21-5.68)$

Montreal L3 disease

$1.98(1.01-3.88)$

Sensitivity analysis (Rx $>1990)$

UC (all cases $=178)$

Montreal E3 disease

$2.26(1.11-4.60)$

Steroid use

$2.29(1.41-3.72)$

Anti-TNF use

$0.37(0.15-0.88)$

$\mathrm{CD}($ all cases $=121)$

Montreal L2 disease

$2.60(1.09-6.20)$

Montreal L3 disease

$2.29(1.08-4.84)$

Anti-TNF use

$0.50(0.27-0.923)$

Melanoma in situ

$\mathrm{UC}($ all cases $=32)$

$\mathrm{CD}($ all cases $=22)$

Age at IBD diagnosis

$1.029(1.007-1.052)$

No risk factors

identified

Sensitivity analysis (Rx $>1990$ )

UC (all cases $=32)$

Age at IBD

diagnosis

$\mathrm{CD}($ all cases $=22)$

Penetrating disease

$1.028(1.003-1.055)$

Anti-TNF use

$3.15(1.08-9.16)$

Malignant melanoma

UC $($ all cases $=146$ )

$\mathrm{CD}($ all cases $=99)$

Montreal E3 disease

$0.13(0.03-0.66)$

Montreal L2 disease

$3.70(1.54-8.90)$

Montreal L3 disease $2.40(1.08-5.31)$

Sensitivity analysis $(\mathrm{Rx}$ $>1990)$

$\mathrm{UC}($ all cases $=146)$

$\mathrm{CD}($ all cases $=99)$

Montreal E3 disease

$3.09(1.53-6.22)$

Montreal L2 disease

Final multivariable regression model after adjustment for duration of follow-up since IBD diagnosis and backward elimination of nonsignificant variables for the identification of independent risk factors to develop melanoma. Similar inclusion periods of IBD diagnosis (since 1991) for cases and controls were used in the sensitivity analysis. Rx: including medication in analysis.

patients with IBD taking thiopurines $(P=0.06$; Fig. 2D) and a better survival of patients using anti-TNF $(P=0.038$; Fig. $2 \mathrm{C})$. In the multivariable analysis, no differences in survival for patients with IBD using anti-TNF were observed (HR: 0.16, 95\% CI, 0.02-1.21). The HR of patients with IBD using thiopurines was 0.55 in the multivariable analysis (95\% CI, 0.25-1.23).

\section{DISCUSSION}

This nationwide cohort study describes risk factors for and the clinical course of melanoma in patients with IBD. The results indicate that a more extensive IBD phenotype is a risk factor for 
TABLE 3. Comparison Between Patients with IBD with Melanoma and Melanoma in the General Population

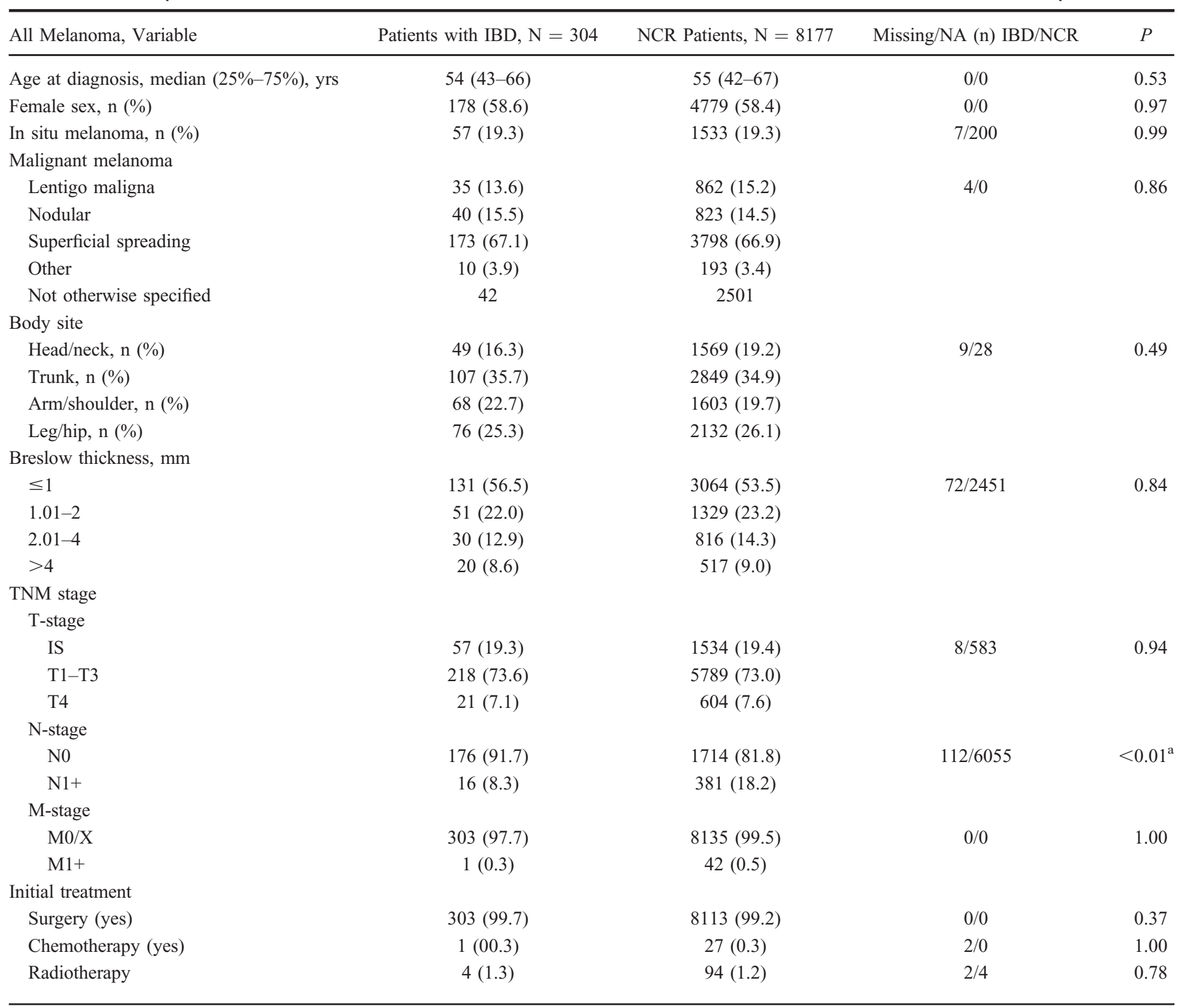

${ }^{\mathrm{a}} P<0.05$.

NCR, Netherlands Cancer Registry.

melanoma development in patients with IBD. Histology, location, and survival of melanoma in patients with IBD are similar compared with the general population. The use of anti-TNF therapy and/or thiopurines did not impair survival after melanoma.

It has been described in other cohorts of immunosuppressed (transplant) patients that survival after melanoma diagnosis is impaired compared with the general population, especially for patients with higher Breslow thickness $(>1.5$ $\mathrm{mm}) .{ }^{10-12,21}$ These data previously suggested the need for treatment adaptation in patients with IBD after a diagnosis of melanoma, by decreasing immune suppression. However, this is not supported by this study, as we found no differences in survival between IBD cases and the general population, also when specifically assessed for immunosuppression. Similar survival results were previously described in a smaller study of patients with IBD $(\mathrm{n}=97),{ }^{22}$ although this study had a longer inclusion period and shorter median follow-up. ${ }^{22}$ However, our results suggesting similar survival between IBD and non-IBD melanoma patients must be interpreted with caution because of a number of reasons. First, the differences with studies in transplant patients may be explained by the limited number of 

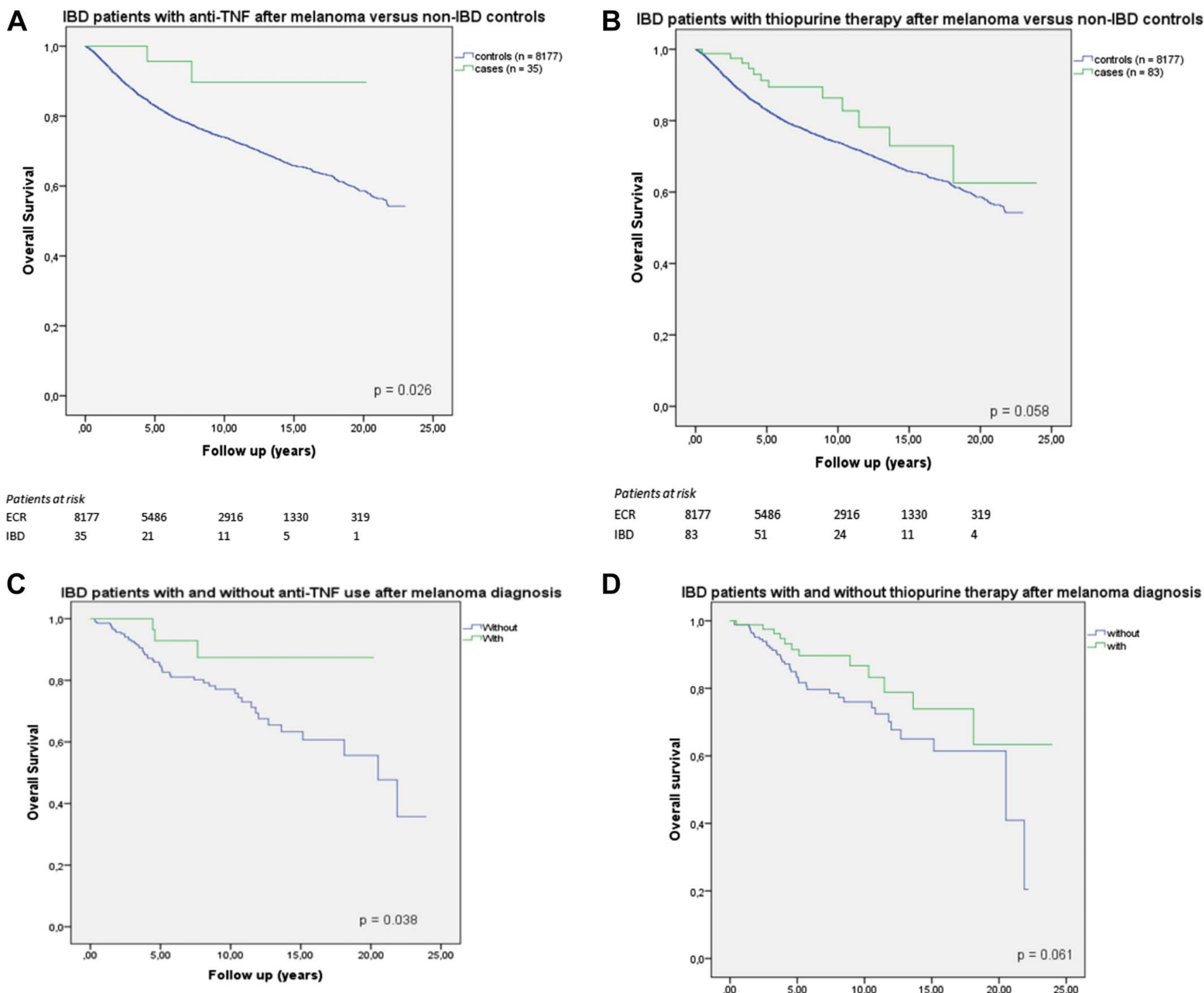

D IBD patients with and without thiopurine therapy after melanoma diagnosis

\begin{tabular}{lllllll}
\multicolumn{2}{l}{ Patients at risk } & & & & & \\
With & 44 & 25 & 12 & 5 & 1 & 0 \\
Without & 223 & 128 & 61 & 24 & 7 & 0
\end{tabular}

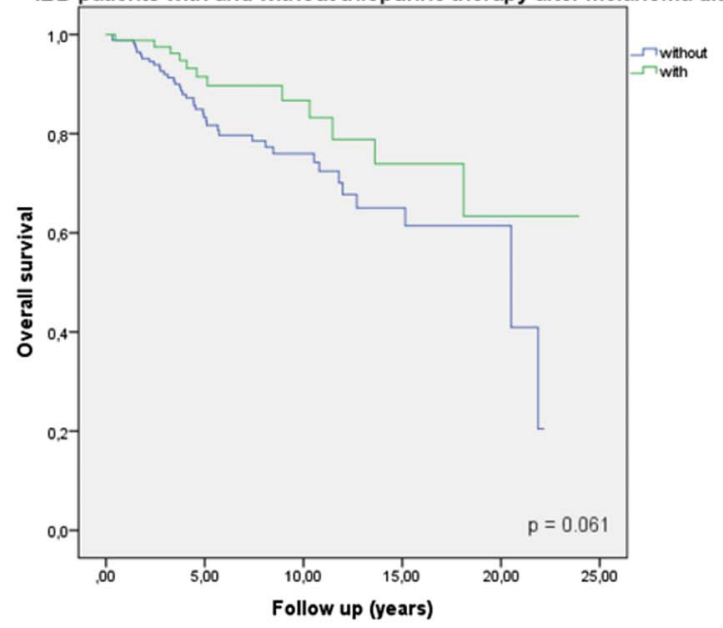

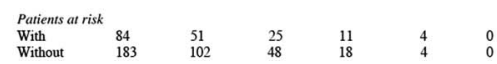

FIGURE 2. Survival analysis. A, Kaplan Meier survival curve: patients with IBD using anti-TNF after melanoma versus non-IBD controls. B, Kaplan Meier survival curve: patients with IBD using thiopurine therapy after melanoma versus non-IBD controls. C, Kaplan Meier survival curve: patients with IBD with and without anti-TNF use after melanoma diagnosis. D, Kaplan Meier survival curve: patients with IBD with and without thiopurine therapy after melanoma diagnosis.

patients with IBD developing a melanoma and by the lower doses, shorter treatment duration and different combinations of immunosuppressive therapy in patients with IBD versus transplant patients. Most patients with IBD will discontinue or switch immunosuppressive therapy, mainly because of side effects or loss of response, ${ }^{23}$ while transplant patients are in general lifelong on immunosuppressive therapy. Moreover, a significant percentage of patients with IBD is treated without immunosuppressive therapy. ${ }^{24}$ Second, Penn ${ }^{25}$ showed that the risk of recurrence is highest in the first 2 years after malignancy diagnosis in (renal) transplant patients. As the moment of starting immunosuppressive therapy after melanoma diagnosis varied widely in our study, this may have also influenced our data, also because it was not possible in this study to specify exactly when patients started or stopped certain immunosuppressive medication, because of the retrospective study design. Third, our finding that thiopurine or anti-TNF therapy did not affect survival may be explained by a selection bias: the fear of a (faster) recurrence implies that these treatments might have been started in patients with a favorable risk profile only. 
IBD extent, including pancolitis and ileocolonic involvement was found to be a risk factor for melanoma development. IBD extent could be a risk factor because more extensive immune activation may be present, which might contribute to melanoma development. Although IBD extent and medication use are not the same, IBD extent could be a surrogate marker for more immunosuppression use, which was difficult to verify during chart reviews and therefore maybe underreported. Because IBDSL data are recorded prospectively, underreporting will occur less likely. This might also explain why anti-TNF therapy is even found as a protective factor in the sensitivity analysis.

This study has several limitations. To address our hypothesis, we used 3 different databases that were constructed in different ways. Our data search is for example retrospective, while the IBDSL, PALGA, and the NCR collect data prospectively. The IBDSL cohort only included patients diagnosed since 1991. Our PALGA study group includes patients with diagnosis before this time and also the total follow-up since diagnosis of IBD is longer. This may have resulted in different treatment regimes because of differences in time frame. Underreporting of immunosepressiva in our retrospective PALGA cohort can be an explanation for the results. Because IBDSL data are recorded prospectively, underreporting will occur less likely in this IBD cohort. Unfortunately, there is no single database available that could answer our research questions sufficiently.

We performed a sensitivity analyses in which only medication of patients with an IBD diagnosis after 1990 was included. Follow-up was included as fixed factor in the analyses to correct for differences in follow-up duration, which were caused by differences in inclusion period. Furthermore, we were not informed about skin type, number of sun burns and melanoma mitotic index because it was not included in the registries and was often not recorded in the medical charts. Finally, for a number of variables in the analyses, there are considerable numbers of missing values. For these variables, results should be interpreted cautiously.

The major strength of this study is the large cohort of IBD and melanoma patients, showing reassuring data on survival. This can influence clinical decision making for patients with IBD with melanoma in daily practice, especially with the current rising incidence and prevalence of both IBD and melanoma. For patients with IBD who develop or had melanoma in the past, treatment decisions still require close collaboration between gastroenterologists, dermatologists, and oncologists, and they must be based on a thorough knowledge of the individual case, including the activity of the IBD, concomitant therapy and melanoma stage. ${ }^{2,26}$

In conclusion, this first study on the clinical course of melanoma in IBD shows no differences in the clinical characteristics of melanoma in patients with IBD and the general population, besides a lower N-stage in patients with IBD. The overall survival and survival in patients with IBD treated with thiopurine or anti-TNF therapy was not impaired. Although these survival data from our nationwide cohort are reassuring for daily practice, treatment choices remain dependent on individual risk assessment by treating physicians and patients.

\section{ACKNOWLEDGMENTS}

The authors would like to thank Prof. Dr. L.A.L.M. Kiemeneij for his epidemiological and statistical advice.

\section{REFERENCES}

1. Pedersen N, Duricova D, Elkjaer M, et al. Risk of extra-intestinal cancer in inflammatory bowel disease: meta-analysis of population-based cohort studies. Am J Gastroenterol. 2010;105:1480-1487.

2. Annese V, Beaugerie L, Egan L, et al. European evidence-based consensus: inflammatory bowel disease and malignancies. J Crohns Colitis. 2015;9:945-965.

3. Long MD, Martin CF, Pipkin CA, et al. Risk of melanoma and nonmelanoma skin cancer among patients with inflammatory bowel disease. Gastroenterology. 2012;143:390-399.e1.

4. Singh H, Nugent Z, Demers AA, et al. Increased risk of nonmelanoma skin cancers among individuals with inflammatory bowel disease. Gastroenterology. 2011;141:1612-1620.

5. van den Heuvel TR, Wintjens DS, Jeuring SF, et al. Inflammatory bowel disease, cancer and medication: cancer risk in the Dutch population-based IBDSL cohort. Int J Cancer. 2016;139:1270-1280.

6. Moran GW, Lim AW, Bailey JL, et al. Review article: dermatological complications of immunosuppressive and anti-TNF therapy in inflammatory bowel disease. Aliment Pharmacol Ther. 2013;38:1002-1024.

7. Peyrin-Biroulet L, Khosrotehrani K, Carrat F, et al. Increased risk for nonmelanoma skin cancers in patients who receive thiopurines for inflammatory bowel disease. Gastroenterology. 2011;141: 1621-1628.e1-5.

8. Singh S, Nagpal SJ, Murad MH, et al. Inflammatory bowel disease is associated with an increased risk of melanoma: a systematic review and meta-analysis. Clin Gastroenterol Hepatol. 2014;12:210-218.

9. Mariette X, Matucci-Cerinic M, Pavelka K, et al. Malignancies associated with tumour necrosis factor inhibitors in registries and prospective observational studies: a systematic review and meta-analysis. Ann Rheum Dis. 2011;70:1895-1904.

10. Russo I, Piaserico S, Belloni-Fortina A, et al. Cutaneous melanoma in solid organ transplant patients. G Ital Dermatol Venereol. 2014;149: 389-394.

11. Penn I. Tumors after renal and cardiac transplantation. Hematol Oncol Clin North Am. 1993;7:431-445.

12. Frankenthaler A, Sullivan RJ, Wang W, et al. Impact of concomitant immunosuppression on the presentation and prognosis of patients with melanoma. Melanoma Res. 2010;20:496-500.

13. Brewer JD, Christenson LJ, Weaver AL, et al. Malignant melanoma in solid transplant recipients: collection of database cases and comparison with surveillance, epidemiology, and end results data for outcome analysis. Arch Dermatol. 2011;147:790-796.

14. Casparie M, Tiebosch AT, Burger G, et al. Pathology databanking and biobanking in The Netherlands, a central role for PALGA, the nationwide histopathology and cytopathology data network and archive. Cell Oncol. 2007;29:19-24.

15. Derikx LA, Kievit W, Drenth JP, et al. Prior colorectal neoplasia is associated with increased risk of ileoanal pouch neoplasia in patients with inflammatory bowel disease. Gastroenterology. 2014;146:119-128.e1.

16. Derikx LA, Nissen LH, Drenth JP, et al. Better survival of renal cell carcinoma in patients with inflammatory bowel disease. Oncotarget. 2015;6:38336-38347.

17. Nissen LH, Assendorp EL, van der Post RS, et al. Impaired gastric cancer survival in patients with inflammatory bowel disease. $J$ Gastrointestin Liver Dis. 2016;25:431-440.

18. Bernstein CN, Blanchard JF, Rawsthorne P, et al. Epidemiology of Crohn's disease and ulcerative colitis in a central Canadian province: a population-based study. Am J Epidemiol. 1999;149:916-924.

19. Satsangi J, Silverberg MS, Vermeire S, et al. The Montreal classification of inflammatory bowel disease: controversies, consensus, and implications. Gut. 2006;55:749-753. 
20. van den Heuvel TR, Jonkers DM, Jeuring SF, et al. Cohort profile: the inflammatory bowel disease South Limburg cohort (IBDSL). Int $J$ Epidemiol. 2017;46:e7, 1-9.

21. Dinh QQ, Chong AH. Melanoma in organ transplant recipients: the old enemy finds a new battleground. Australas J Dermatol. 2007;48:199-207.

22. Shu X, Ji J, Sundquist J, et al. Survival in cancer patients hospitalized for inflammatory bowel disease in Sweden. Inflamm Bowel Dis. 2011;17: 816-822.

23. Jharap B, Seinen ML, de Boer NK, et al. Thiopurine therapy in inflammatory bowel disease patients: analyses of two 8-year intercept cohorts. Inflamm Bowel Dis. 2010;16:1541-1549.

24. Dignass A, Eliakim R, Magro F, et al. Second European evidence-based consensus on the diagnosis and management of ulcerative colitis part 1 : definitions and diagnosis. J Crohns Colitis. 2012;6:965-990.

25. Penn I. Effect of immunosuppression on preexisting cancers. Transplant Proc. 1993;25:1380-1382.

26. Beaugerie L. Management of inflammatory bowel disease patients with a cancer history. Curr Drug Targets. 2014;15:1042-1048.

\section{Appendix 1. APPENDIX WORKING GROUP}

\section{A: PALGA Group}

A. F. Hamel (Ommelander Hospital Group, Delfzijl), A. P. Willig (Laurentius Hospital, Roermond), C. Huysentruyt (Máxima Medical Center, Eindhoven), C. Jansen (Medical Spectrum Twente, Enschede), J. E. Boers (Isala, Zwolle), K. Schelfout (Lievensberg Hospital, Bergen op Zoom), M. Keuppens (ZorgSaam ZeeuwsVlaanderen, Terneuzen), M. Nap (Atrium Medical Centre, Heerlen), P. M. Kluin (University Medical Centre Groningen, Groningen), R. H. J. A Sinke (Groene Hart Hospital, Gouda), R. Riedl (Maastricht University Medical Center, Maastricht), S. H. Sastrowijoto (Orbis Medical Centre, Sittard), H. V. N. Kusters - Vandevelde (Canisius-Wilhelmina Hospital, Nijmegen), R. Natté (Haga Hospital, Den Haag), and C. M. van Dijk (Groene Hart Hospital, Gouda).

\section{B: IBD and Melanoma Group}

A. A. van Bodegraven (Vrije University Medical Centre, Amsterdam), A. C. I. T.L Tan (Canisius Wilhelmina Hospital, Nijmegen), J. J. Meeuse (Hospital Rivierenland, Tiel), A. E. van der Meulen-de Jong (Leiden University Medical Centre, Leiden), B. Oldenburg (University Medical Centre Utrecht, Utrecht), B. C. A. J. Loffeld (Zuwe Hofpoort Hospital, Woerden), B. M. Durfeld (Hospital Group Twente, Almelo), C. J. van der Woude (Erasmus Medical Centre, Rotterdam), D. L. Cahen (Amstelland Hospital, Amstelveen), C. Y. Ponsioen (Academic Medical Centre, Amsterdam), D. Janik (Ommelander Hospital Group, Delfzijl), W. G. M. Mares (Hospital Gelderse Vallei, Ede), L. P. L. Gilissen (Catharina Hospital, Eindhoven), F. L. Wolters (VieCuri, Venlo),
G. Dijkstra (University Medical Centre Groningen, Groningen), G. W. Erkelens (Gelre Hospital, Apeldoorn), Dr. T. J. Tang (IJsselland Hospital, Capelle aan den IJssel), H. G. T. Lam (Diakonessenhuis, Utrecht), H. J. T. Smalbraak (Lievensberg Hospital, Bergen op Zoom), J. C. Thijs (Hospital Bethesda, Hoogeveen), J. H. Voskuil (Hospital Tjongerschans, Heerenveen), J. P. Kuyvenhoven (Kennemer Gasthuis, Haarlem), J. Vecht (Isala, Zwolle), J. J. van Gulick (Amphia Hospital, Breda), J. M. Janssen (Onze Lieve Vrouwe Gasthuis, Amsterdam; BovenIJ Hospital, Amsterdam), J. T. Sarneel (Admiraal De Ruyter Hospital, Middelburg), J. W. M. Tjhie-Wensing (Elkerliek Hospital, Helmond), J. Y. L. Lai (Groene Hart Hospital, Gouda; Langeland Hospital, Zoetermeer), L. T. Vlasveld (Hospital Bronovo, Den Haag), L. E. Oostenbrug (Atrium Medical Centre, Heerlen), M. Gerretsen (Diaconessenhuis Meppel, Meppel), M. E. Bartelink (Deventer Hospital, Deventer), N. Mahmmod (Sint Antonius, Nieuwegein), M. G. V. M. Russel (Medical Spectrum Twente, Enschede), M. J. A. L. Grubben (Sint Elisabeth Hospital, Tilburg), M. K. Vu (Rijnland Hospital, Leiderdorp), M. L. Verhulst (Máxima Medical Centre, Eindhoven), P. Dewint (Van Weel-Bethesda Hospital, Dirksland; Maasstad Hospital, Rotterdam), P. C. F. Stokkers (Sint Lucas Andreas Hospital, Amsterdam), P. J. Bus (Laurentius Hospital, Roermond), P. J. Wismans (Havenziekenhuis, Rotterdam), P. W. E. van der Haeck (Refaja Hospital, Stadskanaal), R. J. L Stuyt (Haga Hospital, Den Haag), R. N. M. Zeijen (Vlietland Hospital, Schiedam), R. P. M Dahlmans (Sint Jans Gasthuis, Weert), S. Vandebosch (ZorgSaam Zeeuws-Vlaanderen, Terneuzen), T. E. H. Romkens (Jeroen Bosch Hospital, Den Bosch), C. Noomen (Medical Center Alkmaar, Alkmaar), W. R. ten Hove (Diaconessenhuis Leiden, Leiden), H. Boot (Antoni van Leeuwenhoek Hospital Amsterdam), K. van der Linde (Medical Center Leeuwarden, Leeuwarden), P. Wahab (Rijnstate Hospital, Arnhem), S.Y. de Boer (Slingeland Hospital, Doetinchem), K. Thurnau (Ropcke Zweers Hospital, Hardenberg) M. van Haastert (Martini Ziekenhuis, Groningen), D. F. G. M. Josemanders (Reinier de Graaf Groep, Delft), R. L. West (Sint Franciscus Gasthuis, Rotterdam), M. J. Pierik (Maastricht University Medical Center, Maastricht), A. C. T. M. Depla (Slotervaart Hospital, Amsterdam), E. T. P. Keulen (Orbis Medical Center, Sittard), W. A. de Boer (Bernhoven Hospital, Uden), A. H. J. Naber (Tergooi Hospital, Hilversum), J. R. Vermeijden (Meander Medical Center, Amersfoort), R. C. Mallant-Hent (Flevo Hospital, Almere), R. Beukers (Albert Schweitzer Hospital, Dordrecht), P. C. J. Ter Borg (Ikazia Hospital, Rotterdam), E. C. R. Halet (Franciscus Hospital, Roosendaal), K. F. Bruin (Twee Steden Hospital, Tilburg), R. K. Linskens (Sint Anna Hospital, Geldrop), and M. T. Uiterwaal (Spaarne Hospital, Hoofddorp). 